\title{
Experimental investigation of strain rate dependent damage mechanisms in DP1000 automotive steel
}

\author{
Sarath Chandran ${ }^{1 *}$, Patricia Verleysen ${ }^{1}$ \\ ${ }^{1}$ MST-DyMaLab, Department of Electromechanical, Systems and Metal Engineering, Faculty of \\ Engineering and Architecture, Ghent University, Technologiepark 46, 9052, Zwijnaarde, Belgium
}

\begin{abstract}
Present study aims to investigate the effect of stress state and loading rates on the damage mechanisms in a DP1000 steel using a welldesigned series of experiments. A specimen family comprising of central hole, in-plane shear and plane strain samples is applied to characterise damage under well-controlled stress states. The optimization of the specimen geometries is achieved using finite element simulations. To assess the influence of strain rate, quasi-static, intermediate and dynamic tests are performed on the designed samples. Local strain fields are obtained by digital image correlation. After testing, scanning electron microscopy is employed to systematically analyse the micromechanisms driving the damage in the investigated material. The underlying damage mechanisms are ferrite-martensite interphase debonding, martensite cracking and debonding at ferrite-ferrite grain boundaries. Stress state and strain rate are found to have distinct influences on triggering the underlying damage mechanisms.
\end{abstract}

Keywords : Dynamic fracture, damage mechanisms, stress state, DP steel, proportional loading, SEM

\section{Introduction}

Over the past decades, the rising demand for light weight structures for enhanced fuel efficiency, concurrently with the goal of achieving efficient passenger safety design in the automotive industry, is met by advanced high strength steels, particularly dual phase (DP) steels [1]. Next to the intrinsic material aspects such as the morphology, volume fraction and spatial size distribution of the phases, deformation behaviour in DP steels is also influenced by strain rate and stress state. The effect of the former aspect has been reasonably wellestablished [2-6], however, the impact of the latter variables on the micromechanisms of deformation is not well-documented. The complex multiphase microstructure of DP steels additionally introduces another level of complexity in the subsequent damage and fracture mechanisms under impact $[7,8]$. Hence, an understanding of the participation of the phase

\footnotetext{
* Corresponding author: Sarath.Chandran@UGent.be
} 
constituents in the deformation and damage processes at static to dynamic strain rates and under multiaxial stress states is essential to establish the relationship between the micro-scale material features and the resultant macro-scale mechanical properties.

Present work reports experimental evidence of the influence of strain rate and stress state on the damage characteristics of a DP1000 steel using mechanical tests performed on 3 purpose-developed fracture specimens at three distinct strain rates. A finite element-based specimen design is incorporated to achieve a constant and distinct stress state in the fracture initiation spot during the entire deformation history. After testing, scanning electron microscopy (SEM) is employed to reveal the principle damage modes.

\section{Material and Methods}

\subsection{Material}

The material used in this investigation is a commercial dual-phase automotive steel (DP1000) that is supplied in the form of rolled sheets with a final thickness of $1.5 \mathrm{~mm}$. The ultra-fine grain microstructure consists of martensite islands $(45 \mathrm{vol} \%)$ dispersed in a ferrite matrix $(55 \mathrm{vol} \%)$.

\subsection{Experimental Methods}

\subsubsection{Test setups and measurement devices}

An Instron 5569 material testing machine is used to characterize the mechanical properties of DP1000 steel at quasi-static and intermediate loading rates. Force measurement is enabled by a calibrated load cell with a maximum capacity of $50 \mathrm{kN}$. Dynamic tests are performed using the split-Hopkinson bar tensile (SHBT) setup of DyMaLab at Ghent University. Here, the test specimen is sandwiched between two long slender bars, i.e., the input and output bar. A tensile loading wave is generated by the direct impact of an impactor on the free end of the input bar. The strain rate in the sample is thus adjusted by controlling the velocity of the impactor. Strain gauge measurements on the bars of the waves allow to calculate the force, relative velocity and displacement imposed on the specimen/bar interfaces.

For all the tests, stereo digital image correlation technique (DIC) is adopted to facilitate full-field 3D displacement and strain measurement on the specimen surface during testing. Commercial MatchID software is used to track the deformation of an applied speckle pattern via a reliability guided algorithm. High resolution images during the static tests are obtained using two 5Mpxl Allied Vision F-033B Stingray cameras together with LED lighting. Two Photron miniAX200 high speed cameras are employed to record images at 75000 fps during intermediate and dynamic tests. The sample surface is illuminated using high-intensity Dedocool lamps. The average speckle size in the range $25-45 \mu$ m enable a spatial resolution of $5-10 \mu \mathrm{m} / \mathrm{pixel}$. The average in-plane displacement and strain resolution is around $90 \mu \mathrm{m}$ and $40 \mu$ strains respectively. For each test condition, three repetitions are performed. The engineering stress is calculated as the ratio of the measured force to the initial cross-section in the central section of the tensile samples, except for the shear sample for which the crosssection of the shear region is applied. Local equivalent plastic strain, $\mathcal{E}_{e q}^{p}$, is defined as:

$$
\varepsilon_{e q}^{p}=\int_{0}^{t} \sqrt{\frac{4}{3}\left[\left(\dot{\varepsilon}_{I}^{p}\right)^{2}+\left(\dot{\varepsilon}_{I}^{p}{\dot{\varepsilon_{I I}}}^{p}\right)+\left({\dot{\varepsilon_{I I}}}^{p}\right)^{2}\right]} d t
$$

Where $\varepsilon_{I}=\ln \left(1+\frac{\partial u_{x}}{\partial X}\right)$ and $\varepsilon_{I I}=\ln \left(1+\frac{\partial u_{y}}{\partial Y}\right)$ are the principal logarithmic strains measured directly on the specimen surface at the necking section or shear region. $\mathrm{X}$ and $\mathrm{Y}$ 
are the reference coordinates along the principal axes, and $u_{x}$ and $u_{y}$ are the corresponding displacements.

\subsubsection{Experimental design for damage and fracture characterization}

To ensure systematic conclusions on the effect of stress state on the damage and fracture of DP1000 steel, a specimen family, including in-plane shear $(\mathrm{SH})$, central hole $(\mathrm{CH})$ and plane strain (PS) specimens, covering a wide range of stress states is carefully designed. A finite element based parametric analysis procedure is performed for geometrical optimization. The design objective is to attain a desired stress state with proportional loading characteristics in the fracture initiation spot during the entire deformation history. The solution of the optimization is ensured via Levenberg-Marquardt algorithm employed in a Python program which actively interacts with the commercial finite element program Abaqus explicit. An isotropic von Mises plasticity model is incorporated in which the hardening behaviour at room temperature for a large strain range is described using a combined Swift-Voce law:

$\sigma_{t r}\left(\varepsilon_{p}\right)=\alpha \cdot A\left(\varepsilon_{0}+\varepsilon_{p}\right)^{n}+(1-\alpha) \cdot\left\{k_{0}+Q\left[1-\exp \left(-\beta_{v} \varepsilon_{p}\right)\right]\right\}$

$\sigma$ and $\mathcal{E}_{p}$ are the true stress and true plastic strain, respectively. A, $\mathcal{E}_{0}, \mathrm{n}, \mathrm{k}_{0}, \mathrm{Q}, \beta_{\mathrm{v}}$ and $\alpha$ are the material parameters. The first term of the equation refers to the Swift law, the second to the Voce law. $\alpha$ is a weighting parameter. The resultant flow curve is shown in Fig 1a.


Fig. 1. (a) Experimental and combined Swift-Voce law-based flow curve. The material constants are: $\mathrm{A}=1305 \mathrm{MPa}, \mathcal{E}_{0}=2.25 \mathrm{e}-14, \mathrm{n}=0.0768, \mathrm{k}_{0}=776.28 \mathrm{MPa}, \mathrm{Q}=265.49 \mathrm{MPa}, \beta_{\mathrm{v}}=74.34$ and $\alpha=0.5$. (b) Loading history in the critical element for the three considered geometries.

The loading paths, i.e. the evolution of the stress state variables, stress triaxiality and Lode angle parameter, with respect to equivalent plastic strain (PEEQ), of the critical element is confirmed to be proportional in Fig 1b. The final specimen family is displayed in Fig 2. Identical samples and boundary conditions are used across all the tests to eliminate test artefacts and also to ensure that differences between the results can be solely assigned to the deformation rate. The DIC displacements used in the result section are measured across the blue dots for all the tested samples as shown in Fig 2.
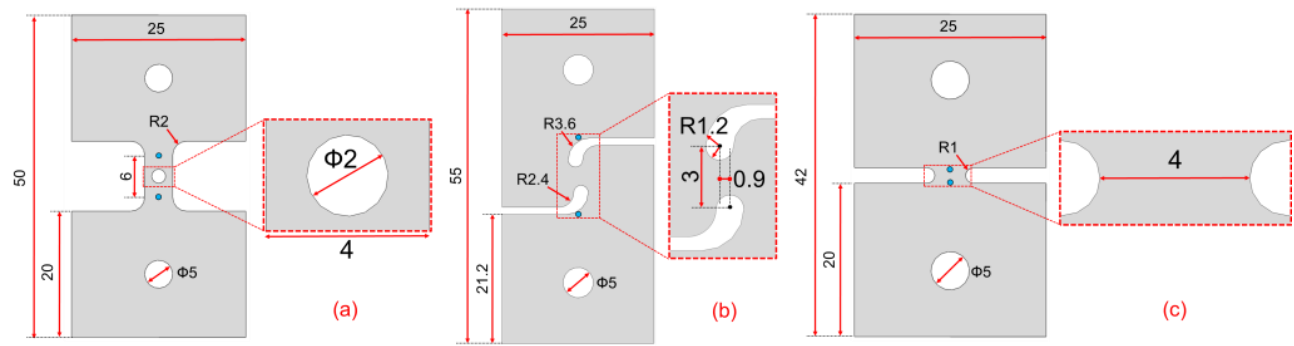

Fig. 2. Specimen geometries: (a) Central hole (b) Shear (c) Plane strain 


\subsubsection{Micro-scale observation}

Scanning electron microscopy (SEM) is employed after testing to analyse the underlying damage mechanisms. The associated micromechanisms driving the damage are investigated using the SEM micrographs of the through-thickness cross-section polished to the critical damage initiation planes. Micro-constituents and phases are revealed by chemical etching with Nital $2 \%$ for $8-10$ s.

\section{Results and discussion}

\subsection{Mechanical behaviour}

With the designed fracture specimens, all the tests are carried out along the specimen's rolling direction. Quasi-static, intermediate and dynamic tests aim at average plastic strain rates of $10^{-4} \mathrm{~s}^{-1}, 1$ and $1000 \mathrm{~s}^{-1}$ in the central gauge section or the shear region of the samples. Fig $3 \mathrm{a}-$ c presents the representative nominal stress-displacement curves of the $\mathrm{CH}, \mathrm{SH}$ and PS samples respectively across the aforementioned strain rate regimes.
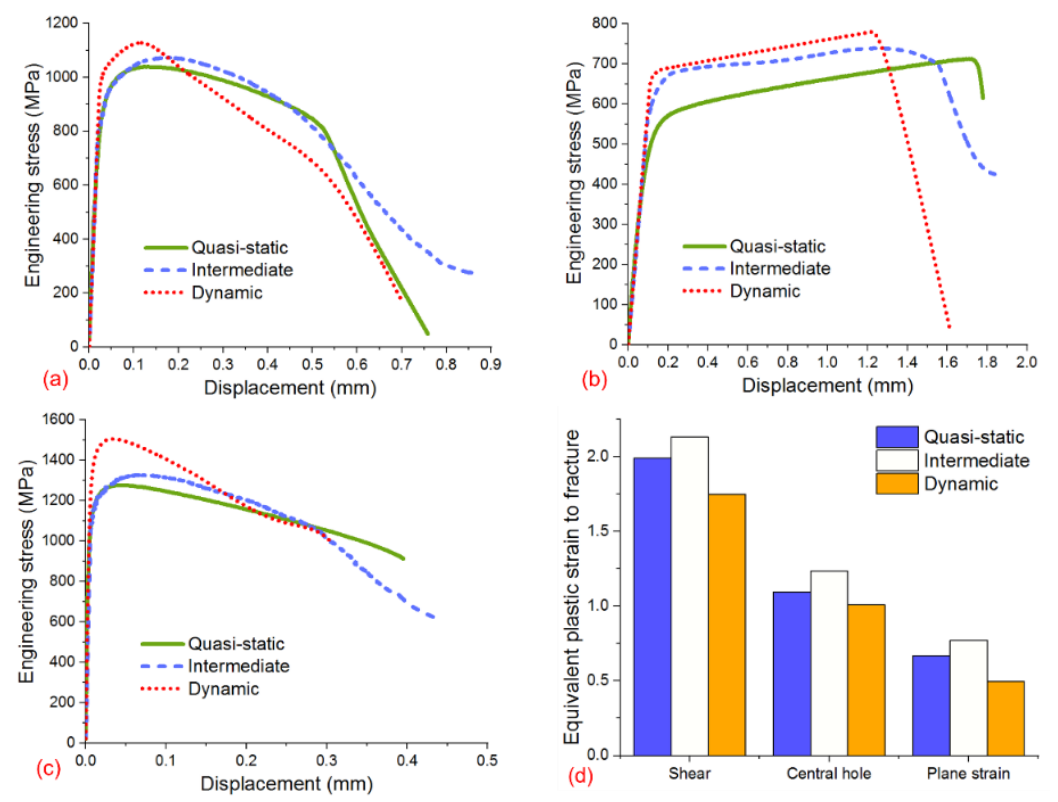

Fig. 3. Engineering stress-displacement plots of (a) central hole (b) shear and (c) plane strain specimens at quasi-static, intermediate and dynamic test speeds. (d) Influence of stress state and strain rate on fracture strain.

It is observed that the mechanical strength is positively affected by the strain rate for all the fracture specimens. Much of the improvement occurs in the transition to the dynamic loading rates for the tensile specimens, whereas for the SH sample, enhanced strength is achieved even from the intermediate strain rate. Going from shear to plane strain, significantly higher work hardening strength is achieved on account of increasing stress triaxiality. However, such material strengthening often comes at the expense of ductility. Negative dependence of the stress state on the equivalent plastic strain to fracture is evident in Fig 3d. Shear stress state effectively postpones damage and fracture occurrence by preventing the occurrence of diffused and localized necking. PS samples with the highest stress triaxiality experience the lowest fracture elongation. Across all the stress states, the strain to fracture is non-monotonically influenced by the strain rate. 


\subsection{Damage mechanisms analysis}

Microstructure-level damage mechanism analysis under different stress states and loading rates is performed by SEM analysis. The fractured samples are ground and polished as close as possible to the critical damage initiation plane where severe plastic deformation occurred. For the $\mathrm{CH}$ specimen, such a location lies at the intersection of the circular hole edge and the mid-transverse direction, and for the other samples at the middle width spot. The damage initiation plane corresponds well with the critical zone in finite element simulations. Hence, the damage mechanisms can be directly linked with the stress state. Through-thickness crosssection micrographs of $\mathrm{CH}, \mathrm{SH}$ and PS samples just below the fracture surface are displayed in Fig $4 \mathrm{a}, \mathrm{b}$ and $\mathrm{c}$ respectively.

(a)

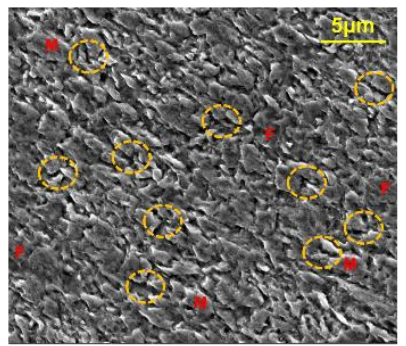

(b)
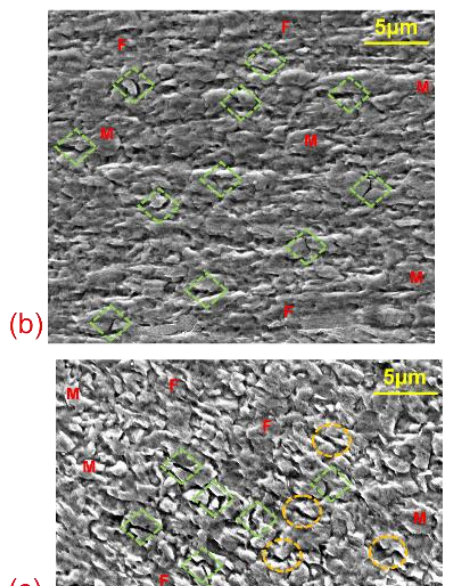

(c)

RD

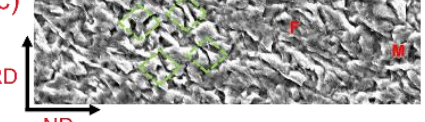

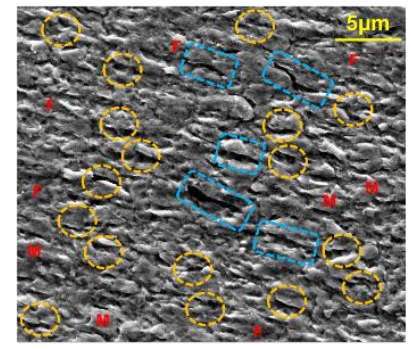
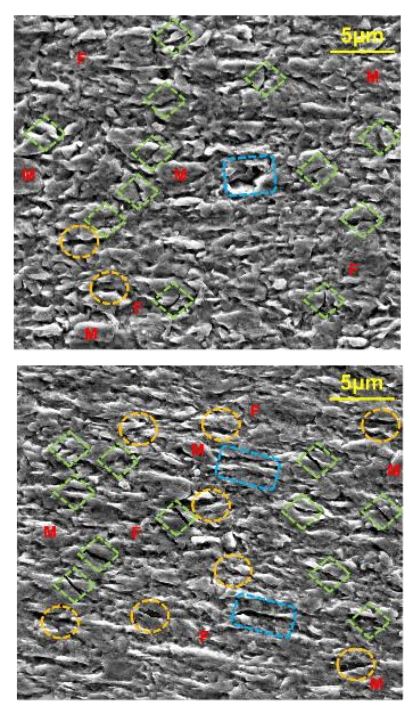
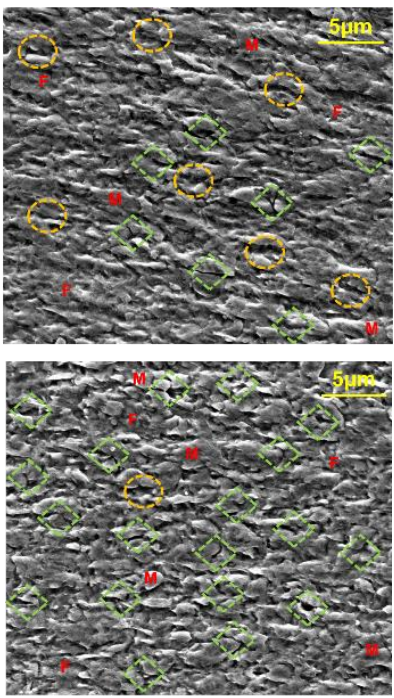



Fig.4 SEM micrographs of (a) $\mathrm{CH}$ (b) SH and (c) PS specimens at quasi-static (left), intermediate (middle) and dynamic (right) testing speeds. Interface debonding, ferrite-ferrite grain boundary decohesion and martensite cracking are indicated with yellow circles, blue rounded rectangles and green diamonds respectively.

The associated micro-mechanisms driving damage are found to be ferrite-martensite interface decohesion, martensite cracking and ferrite-ferrite grain boundary decohesion. In terms of effect of stress state, the central hole specimen under uniaxial stress state exhibits more micro defects that occur as a result of debonding at the original ferrite-martensite interphases. This is replaced by small-scale cracking of the brittle martensite phase at low stress triaxialities under shear stress state. It is evident from the PS specimen micrographs that the dominant micro void initiation mode at higher stress triaxialities is the martensite cracking accompanied by debonding generated at the original ferrite-martensite interface 
between two closed martensite clusters as well as around the interface of ferrite and martensite phase boundaries. Hence, a decreasing Lode angle parameter tends to increase the probability of martensite cracking. In all the samples, an increase in micro-defects as a result of interface debonding is observed from quasi-static to intermediate loading rates, whereas dynamic testing speeds diminish such an effect and trigger more brittle martensite cracking thus promoting early fracture. In addition to the aforementioned micromechanisms, decohesion of ferrite-ferrite grain boundary is observed for all the samples at intermediate strain rate. The debonding originates at the ferrite-martensite phase, then propagates to the interior of ferrite grains resulting in a crack formation. Such an effect could be attributed to dislocation sub-cell formation in ferrite by virtue of deformation gradients from the interface to the grain interior. Combination of these micromechanisms delays failure at intermediate loading rate.

\section{Conclusions}

With the aim of elucidating the effect of strain rate on the mechanical behaviour and damage mechanisms in a DP1000 steel, lab-scale tests were performed under explicitly defined stress states and deformation rates. Increase in strain rate leads to an improvement in work hardening strength. Such an enhancement was further assisted by increasing stress triaxiality. Contrary to the strength, the ductility is detrimentally affected by the stress state. However, strain rate imparts a non-monotonic fracture strain response. The mechanisms of damage in DP1000 steel vary with both stress state and strain rate. Ferrite-martensite interface debonding, active at higher stress triaxialities, is effectively replaced by martensite cracking at low stress triaxialities. Interface debonding, martensite cracking and ferrite-ferrite grain boundary debonding co-exist at intermediate strain rate and hence, delay the fracture. Martensite cracking is activated under low Lode angle parameter and dynamic strain rates. The latter, however, severely diminishes the deformation capacity of the DP1000 steel. A direct relationship between the imparted stress state and the underlying deformation micromechanisms thus facilitates the engineering of advanced steels.

The authors gratefully acknowledge the European Commission Research Fund for Coal and Steel (RFCS) [grant number : 709711] for the financial funding for the project "Toolkit for the design of damage tolerant microstructures" which supports the investigations and the project partners for extensive collaboration.

\section{References}

1. S. Keeler, M. Kimchi, Advanced high strength steel application guidelines version 5.0, World Auto Steel (2014).

2. S.K Paul, Mater.Des. 44, 397-406 (2013).

3. J. Kadhodapour, A.Butz, S.Ziaei-Rad, Acta Mater. 59(7), 2575-2588 (2011a).

4. C.C Tasan, J.P.M. Hoefnagels, M.G.D. Geers, Acta Mater. 57(17), 4957-4966 (2009).

5. E. Ahmed, T. Manzoor, K.L. Ali, J. Akhter, J. Mater. Eng. Perform. 9(3), 306-310 (2000).

6. X. Sun, K.S. Choi, W.N. Liu, M.A. Khaleel, Int. J. Plast. 25(10),1888-1909 (2009).

7. M. Xu, H. Li, R.T. Jiang, D. Tang, H.T. Jiang, Z.L. Mi, J. Iron Steel Res. Int. 26, 173181 (2019).

8. A. Das, M. Ghosh, S. Tarafder, S. Sivaprasad, D. Chakrabarti, Mater. Sci. Eng. A. 680, 249-258 (2017). 